\title{
Transient free convection in an inclined square porous cavity filled with a nanofluid using LTNE and Buongiorno's models
}

\author{
Mikhail Sheremet ${ }^{1}$, and Ioan Pop ${ }^{2, *}$ \\ ${ }^{1}$ Laboratory on Convective Heat and Mass Transfer, Tomsk State University, 36 Lenin Avenue, 634050 Tomsk, Russia \\ ${ }^{2}$ Department of Mathematics, Babeș-Bolyai University, 400084 Cluj-Napoca, Romania
}

\begin{abstract}
The combined effect of Brownian diffusion, thermophoresis and cavity inclination angle on natural convective heat transfer in an inclined porous enclosure has been studied numerically. Fluid containing nanoparticles of low concentration circulates inside the cavity under the effect of the buoyancy force. Governing equations with corresponding boundary conditions formulated using the non-dimensional stream function and vorticity variables have been solved by the finite difference method. An influence of the cavity inclination angle, Darcy and Nield numbers on nanofluid flow and heat transfer has been investigated. It has been found that high Nield numbers illustrate more equilibrium temperature distribution inside the porous cavity.
\end{abstract}

\section{Introduction}

Fluid flow in porous media has been an area of intensive investigation for the last decades. The growing emphasis on effective granular and fibrous insulation systems, for the successful containment of the transport of radionuclide from deposits of nuclear waste materials, has stimulated various studies in fluid saturated porous media and many results were obtained for the forced and convective flow in the fundamental geometries of internal (cavities, annulus, etc.) and external (over surfaces) flows (Nield and Bejan [1]). Comprehensive reviews of heat transfer mechanisms in geothermal systems has been first presented by Cheng [2,3], and Bejan [4] with emphasis on its applications in geothermal and energy systems research. Since then a large number of practical applications, both industrial and environmental, have caused a rapid extension of the research, and a substantial number of articles, which relate the boundary-layer flow past surfaces of various flow configuration models, have been published. Nield and Bejan [1], Ingham and Pop [5-7], Pop and Ingham [8], Vafai $[9,10]$ and Bejan et al. [11] identified many applications that highlight the directions where further theoretical and experimental developments and investigations are required.

The aim of the present study is to analyze numerically transient free convection in an inclined square porous cavity filled with a water-based nanofluid using LTNE and Buongiorno's models. The partial differential equations with corresponding initial and boundary conditions have been solved using a developed in-house computational fluid dynamics code on the basis of the finite difference method of the second order accuracy.

\section{Analysis and modelling}

A two-dimensional inclined square porous cavity with sides of length $L$, filled with a water-based nanofluid is considered (Fig. 1). The bottom horizontal wall has a local zone of constant hot temperature $\left(T_{h}\right)$ with length $d$, while the remainder of this wall is adiabatic. The left and right vertical walls are maintained at constant cold temperature $\left(T_{c}\right)$, where $T_{h}>T_{c}$, while the top horizontal wall is adiabatic. The following assumptions are made in the study:

(a) The Darcy model and the Boussinesq approximation are applicable.

(b) Nanofluid and the solid matrix of the porous medium are not in local thermodynamic equilibrium.

(c) The properties of the nanofluid and the porous medium are homogeneous and isotropic with permeability $K$.

(d) The base fluid physical properties are constant except for its density.

(e) The boundaries of the cavity are assumed to be impermeable.

(f) The local thermal equilibrium model is also employed to account the temperature difference between the base fluid and nanoparticles.

(g) Nanoparticles are well suspended in the nanofluid by using either surfactant or surface charge technology that prevents the nanoparticles from agglomeration and deposition on the porous matrix.

The equations governing the unsteady Darcy free convection flow of the nanofluid using the mathematical nanofluid model proposed by Bungiorno $[12,13]$ and with the above mentioned assumptions can be written in the form (see Nield and Bejan [1]), including the effects

\footnotetext{
* Corresponding author: popm.ioan@yahoo.co.uk, ipop@math.ubbcluj.ro
} 
of porous matrix, Brownian diffusion, thermophoresis and heat transfer between porous matrix and nanofluid, while nanoparticles and the base fluid are in the local thermal equilibruim.

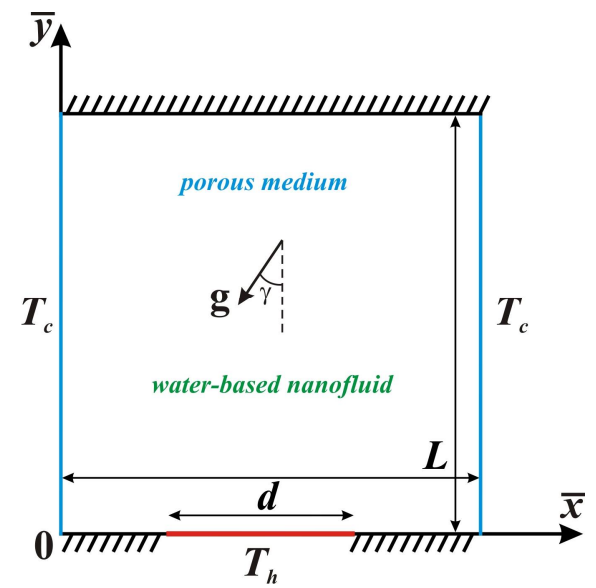

Fig. 1. Physical model together with the coordinate system.

Taking into account the dimensionless stream function and vorticity variables as well as above-mentioned approaches, the governing equations can be formulated in the following form [12]:

$$
\begin{aligned}
& \frac{\partial^{2} \psi}{\partial x^{2}}+\frac{\partial^{2} \psi}{\partial y^{2}}=D a \sqrt{\frac{R a}{P r}}\left\{\frac{\partial \theta_{f}}{\partial y} \sin (\gamma)-\frac{\partial \theta_{f}}{\partial x} \cos (\gamma)\right\} \\
& +D a \sqrt{\frac{R a}{P r}} N r\left\{\frac{\partial \phi}{\partial x} \cos (\gamma)-\frac{\partial \phi}{\partial y} \sin (\gamma)\right\} \\
& \varepsilon \frac{\partial \theta_{f}}{\partial \tau}+\frac{\partial \psi}{\partial y} \frac{\partial \theta_{f}}{\partial x}-\frac{\partial \psi}{\partial x} \frac{\partial \theta_{f}}{\partial y}=\frac{\varepsilon}{\sqrt{R a \cdot P r}}\left(\frac{\partial^{2} \theta_{f}}{\partial x^{2}}+\right. \\
& \left.+\frac{\partial^{2} \theta_{f}}{\partial y^{2}}\right)+\frac{\varepsilon \cdot N b}{\sqrt{R a \cdot P r}}\left(\frac{\partial \phi}{\partial x} \frac{\partial \theta_{f}}{\partial x}+\frac{\partial \phi}{\partial y} \frac{\partial \theta_{f}}{\partial y}\right)+ \\
& +\frac{\varepsilon \cdot N t}{\sqrt{R a \cdot P r}}\left[\left(\frac{\partial \theta_{f}}{\partial x}\right)^{2}+\left(\frac{\partial \theta_{f}}{\partial y}\right)^{2}\right]+\frac{H}{\sqrt{R a \cdot P r}}\left(\theta_{s}-\theta_{f}\right) \\
& \frac{\partial \theta_{s}}{\partial \tau}=\frac{\alpha_{s} / \alpha_{f}}{\sqrt{R a \cdot P r}}\left(\frac{\partial^{2} \theta_{s}}{\partial x^{2}}+\frac{\partial^{2} \theta_{s}}{\partial y^{2}}\right)+ \\
& +\frac{H \xi}{\sqrt{R a \cdot P r}}\left(\theta_{f}-\theta_{s}\right) \\
& \varepsilon \frac{\partial \phi}{\partial \tau}+\frac{\partial \psi}{\partial y} \frac{\partial \phi}{\partial x}-\frac{\partial \psi}{\partial x} \frac{\partial \phi}{\partial y}=\frac{\varepsilon}{L e \sqrt{R a \cdot P r}}\left(\frac{\partial^{2} \phi}{\partial x^{2}}+\right. \\
& \left.+\frac{\partial^{2} \phi}{\partial y^{2}}\right)+\frac{\varepsilon}{L e \sqrt{R a \cdot P r}} \frac{N t}{N b}\left(\frac{\partial^{2} \theta_{f}}{\partial x^{2}}+\frac{\partial^{2} \theta_{f}}{\partial y^{2}}\right)
\end{aligned}
$$

Following the geometry in Fig. 1, we assume that the initial and boundary conditions for these equations are $\psi=0, \theta_{f}=\theta_{s}=0$ at $\tau=0$ $\psi=0$ on the walls of the cavity $\theta_{f}=\theta_{s}=0, N b \partial \phi / \partial x+N t \partial \theta_{f} / \partial x=0$ at $x=0,1$ $\frac{\partial \theta_{f}}{\partial y}=\frac{\partial \theta_{s}}{\partial y}=\frac{\partial \phi}{\partial y}=0$ at $y=0$ outside the heater

$\theta_{f}=\theta_{s}=1, N b \frac{\partial \phi}{\partial y}+N t \frac{\partial \theta_{f}}{\partial y}=0$ at $y=0$ at the heater

$\frac{\partial \theta_{f}}{\partial y}=\frac{\partial \theta_{s}}{\partial y}=\frac{\partial \phi}{\partial y}=0$ at $y=1$

Here $R a$ is the Rayleigh number, $N r$ is the buoyancy ratio parameter, $N b$ is the Brownian motion parameter, $N t$ is the thermophoresis parameter and $L e$ is the Lewis number, which are defined as

$$
\begin{aligned}
& R a=\frac{\rho_{f} g \beta \Delta T\left(1-C_{0}\right) L^{3}}{\mu \alpha_{f}}, N r=\frac{\left(\rho_{p}-\rho_{f}\right) C_{0}}{\left(1-C_{0}\right) \rho_{f} \beta \Delta T}, \\
& P r=\frac{\mu}{\rho_{f} \alpha_{f}}, D a=\frac{K}{L^{2}}, N b=\frac{\sigma D_{B} C_{0}}{\alpha_{f}}, L e=\frac{\alpha_{f}}{D_{B}}, \\
& N t=\frac{\sigma D_{T} \Delta T}{\alpha_{f} T_{c}}, H=\frac{L^{2} h}{k_{f}}, \xi=\frac{(\rho c)_{f}}{(\rho c)_{s}(1-\varepsilon)}
\end{aligned}
$$

The physical quantities of interest are the local fluid and solid Nusselt numbers $N u_{f}$ and $N u_{s}$ evaluated at the heated part of the bottom wall, which are defined, respectively, by

$$
N u_{f}=-\left.\frac{\partial \theta_{f}}{\partial y}\right|_{y=0}, N u_{s}=-\left.\frac{\partial \theta_{s}}{\partial y}\right|_{y=0}
$$

and the average Nusselt numbers $\overline{N u_{f}}$ and $\overline{N u_{s}}$ evaluated at the heated part of the bottom wall, which are given by

$$
\overline{N u_{f}}=\frac{1}{D} \int_{(1-D) / 2}^{(1+D) / 2} N u_{f} d x, \overline{N u_{s}}=\frac{1}{D} \int_{(1-D) / 2}^{(1+D) / 2} N u_{s} d x
$$

Equations (1)-(4) with corresponding initial and boundary conditions were solved using an in-house computational code developed by means of the finite difference method of the second order accuracy [12, 14, 15]. The developed model and numerical method have been verified comprehensively using the numerical and experimental data of other authors [12, 14-17].

The grid independency test has been performed for the present formulation (see Fig. 1) at $R a=10^{5}$, $\operatorname{Pr}=6.82, \quad D a=10^{-3}, \quad \varepsilon=0.5, \quad N r=5, \quad N b=10^{-6}$, $N t=10^{-6}, L e=1000, \alpha_{s} / \alpha_{f}=649.7, \quad \xi=3.4, \quad H=10$. During analysis the porous medium is the aluminum foam. Four uniform grids are tested. Table 1 presents an influence of the grid elements on the average Nusselt numbers.

Table 1. Effect of mesh on the average Nusselt numbers.

\begin{tabular}{|c|c|c|}
\hline $\begin{array}{c}\text { Uniform } \\
\text { mesh }\end{array}$ & $\overline{N u_{f}}$ & $\overline{N u_{s}}$ \\
\hline $200 \times 200$ & 4.63 & 1.88 \\
\hline $300 \times 300$ & 5.77 & 1.89 \\
\hline $400 \times 400$ & 5.94 & 1.90 \\
\hline
\end{tabular}


Using the results presented in Table 1, the uniform grid of $300 \times 300$ points has been chosen for the present study.

\section{Results and Discussion}

Analysis has been carried out for the following values of the governing parameters: $R a=10^{5}, \quad P r=6.82$, $D a=10^{-5}-10^{-3}, \varepsilon=0.5, H=10-1000, N r=5, N b=10^{-6}$, $N t=10^{-6}, L e=1000, \alpha_{s} / \alpha_{f}=649.7, \xi=3.4, \gamma=0-\pi / 2$. Particular efforts have been focused on the effects of the Darcy number, Nield number and cavity inclination angle on streamlines, isotherms, isoconcentrations and average Nusselt numbers for the nanofluid and solid matrix.

Figure 2 shows streamlines, isotherms for nanofluid and porous matrix and isolines of nanoparticles concentrations inside the cavity for $D a=10^{-3}, \gamma=0$ and different values of the Nield number.
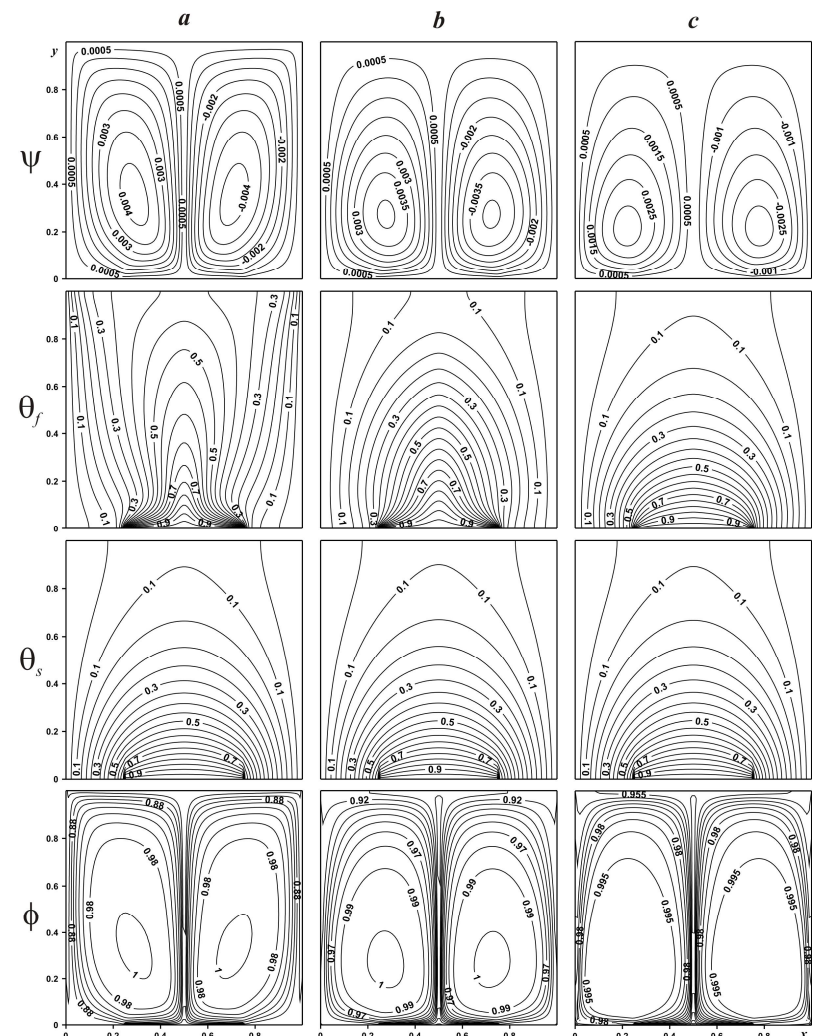

Fig. 2. Streamlines $\psi$, nanofluid isotherms $\theta_{n f}$, solid matrix isotherms $\theta_{s}$ and isolines of nanoparticles volume fraction $\phi$ at $D a=10^{-3}, \gamma=0$ for: $H=10-a, H=100-b, H=1000-c$.

It should be noted that the Nield number characterizes the interface heat transfer between the nanofluid/solidmatrix phases. Low values of the Nield number illustrate weak thermal interaction between the fluid phase and solid matrix and as a result, one can find more intensive circulation inside the cavity with a stable thermal plume formed over the local heater $(D=d / L=0.5)$. Regardless of the Nield number values two convective cells are appeared within the cavity with clockwise and counterclockwise motions. It is worth noting that intensive circulation is characterized by upper position of the convective cells cores, while an attenuation of the convective flow leads to a reduction of the convective cells cores up to the bottom wall. Growth of the Nield number illustrates more intensive thermal interaction between the fluid phase and solid matrix that results in a domination of the heat conduction (isotherms of the nanofluid become similar to the solid matrix isotherms for high values of $H$ ). Convective fluid rate decreases with the Nield number and distributions of nanoparticles become more homogeneous with low differences in $\phi$ inside the cavity, while high differences in $\phi$ can be found near the walls.

Figure 3 demonstrates the average Nusselt numbers at the heater for the cases of the fluid phase $(a)$ and solid matrix $(b)$ in dependences on the dimensionless time and the Nield number. It should be noted that $\tau=300$ can be considered as a steady state, while for $H=10$ one can find small changes in $\overline{N u_{f}}$ with $\tau$. As has been mentioned above, low values of $H$ reflect weak interaction between fluid and solid and as a result $\overline{\mathrm{Nu}}$ is high, while a rise of the Nield number leads to the reduction of the heat transfer rate inside the nanofluid. At the same time, $\overline{N u_{s}}$ decreases also with $H$. Further growth of the Nield number can lead to the equality of the average Nusselt numbers for the fluid phase and solid matrix.

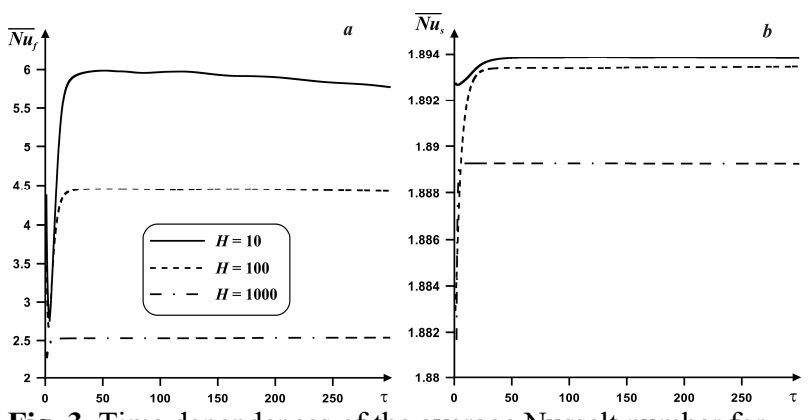

Fig. 3. Time dependences of the average Nusselt number for nanofluid ( $a$ ) and solid matrix (b) at $D a=10^{-3}, \gamma=0$ and different values of the Nield number.

Effects of the cavity inclination angle, Darcy and Nield numbers on the average Nusselt numbers for the fluid phase and solid matrix are shown in Figs. 4 and 5.

Figure 4 illustrates an influence of the cavity inclination angle and the Darcy number on the heat transfer rate in the nanofluid and solid matrix. Low values of the Darcy number characterize a porous medium with low permeability, therefore a growth of $D a$ leads to the heat transfer enhancement inside the fluid phase. At the same time, the average Nusselt number inside the solid matrix has non-linear behavior with $D a$. A growth of the cavity inclination angle between 0 and $\pi / 2$ results in the heat transfer rate reduction inside the fluid phase for high values of the Darcy number, while for low values of $D a$ a raise of $\gamma$ leads to weak changes of $\overline{N u_{f}}$. It is interesting to note that $\overline{N u_{s}}$ diminishes with $\gamma$ for $H=10$, while for $H=100$ and $H=1000 \overline{N_{s}}$ increases with the cavity inclination angle. 


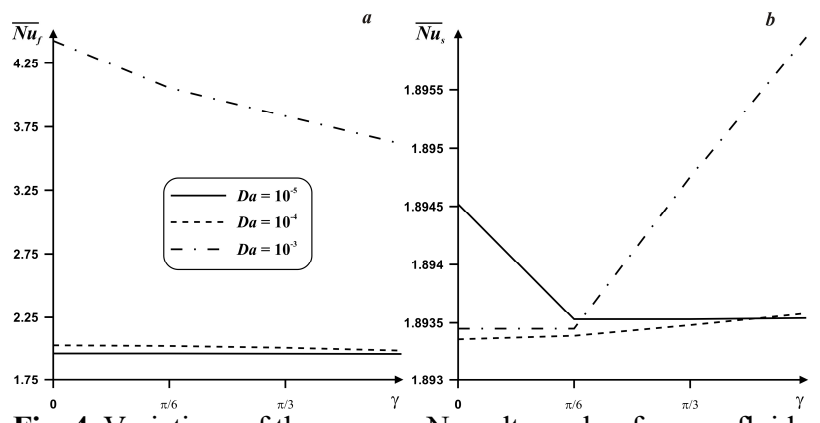

Fig. 4. Variations of the average Nusselt number for nanofluid (a) and solid matrix $(b)$ for $H=100$ and different values of the cavity inclination angle and Darcy number.

Figure 5 demonstrates variations of the average Nusselt numbers with the cavity inclination angle and the Nield number for $D a=10^{-4}$. It should be noted that for $H=10$ a growth of $\gamma$ from 0 till $\pi / 3$ leads to the heat transfer enhancement inside the nanofluid, while for other values of $H \overline{N u_{f}}$ decreases with $\gamma$. A raise of the Nield number leads to the average Nusselt number increase in the nanofluid phase, while $\overline{N u_{s}}$ decreases with $H$. Such behavior with the Nield number can be exlained by the considered conduction regime for $D a=10^{-4}$.

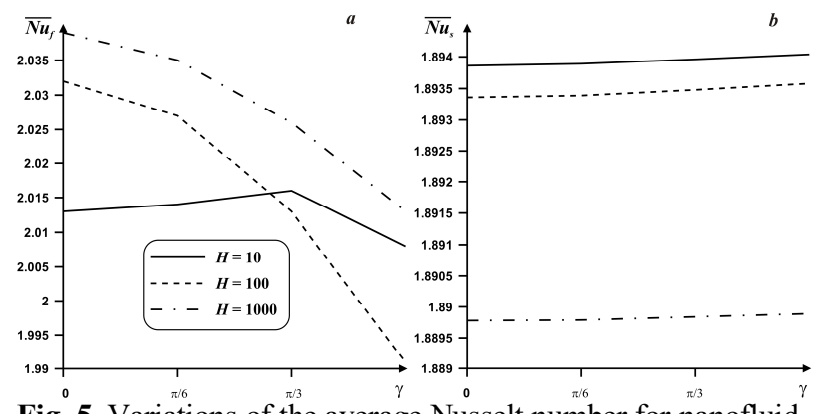

Fig. 5. Variations of the average Nusselt number for nanofluid (a) and solid matrix (b) for $D a=10^{-4}$ and different values of the cavity inclination angle and Nield number.

\section{Conclusions}

Natural convection of nanofluid inside an inclined porous cavity with a local heater on the bottom wall has been studied numerically using the two-phase nanofluid model and local thermal non-equilibrium approach. Particular efforts have been focused on the effects of the cavity inclination angle, Darcy number and Nield number on flow field, temperature and nanoparticles volume fraction distributions as well as average Nusselt numbers at the local heater inside the nanofluid phase and solid matrix. It has been found that for high values of the Darcy number (convective flow and heat transfer regime) a growth of the Nield number leads to the reduction of the heat transfer rate inside the fluid phase. At the same time, $\overline{N u_{f}}$ decreases with the cavity inclination angle also for high $\mathrm{Da}$.

\section{Acknowledgements}

This work of the first author was supported by the Grants Council under the President of the Russian Federation (Project No. MD-2819.2017.8). The work of the second author was supported from the grant PN-III-P4-ID-PCE-2016-0036, UEFISCDI, Romania.

\section{References}

1. D.A. Nield, A. Bejan, Convection in Porous Media ( $4^{\text {th }}$ edition, Springer, New York, 2013).

2. P. Cheng, Adv. Heat Transfer 14 (1978).

3. P. Cheng, In: S. Kakaç, W. Aung, R. Viskanta (eds.) Natural Convection: Fundamentals and Applications. (Hemisphere, Washington, DC, 1985).

4. A. Bejan, In: S. Kakaç, R.K. Shah, W. Aung (eds.), Handbook of Single-Phase Convective Heat Transfer, Chapter 16 (Wiley, New York, 1987).

5. D.B. Ingham, I. Pop, Transport Phenomena in Porous Media (Vol. I, Pergamon, Oxford, 1998).

6. D.B. Ingham, I. Pop, Transport Phenomena in Porous Media (Vol. II, Pergamon, Oxford, 2002).

7. D.B. Ingham, I. Pop, Transport Phenomena in Porous Media (Vol. III, Elsevier, Oxford, 2005).

8. I. Pop, D.B. Ingham, Convective Heat Transfer: Mathematical and Computational Modelling of Viscous Fluids and Porous Media (Pergamon, Oxford, 2001).

9. K. Vafai, Handbook of Porous Media (Marcel Dekker, New York, 2000).

10. K. Vafai, Handbook of Porous Media (Taylor \& Francis, New York, 2005).

11. A. Bejan, I. Dincer, S. Lorente, A.F. Miguel, A.H. Reis, Porous and Complex Flow Structures in Modern Technologies (Springer, New York, 2004).

12. A. Shenoy, M. Sheremet, I. Pop, Convective Flow and Heat Transfer from Wavy Surfaces: Viscous Fluids, Porous Media and Nanofluids (CRC Press, New York, 2016).

13. J. Buongiorno, J. Heat Transfer 128 (2006).

14. M.A. Sheremet, I. Pop, Int. J. Heat Mass Transfer 79 (2014).

15. M.A. Sheremet, I. Pop, Transport in Porous Media 105 (2014).

16. M.Z. Saghir, A. Ahadi, A. Mohamad, S. Srinivasan, Int. J. Thermal Sciences 109 (2016).

17. M.Z. Saghir, A. Mohamad, Int. J. Numerical Methods Heat Fluid Flow 28 (2018). 